\title{
On the resonance excitation of nearly diurnal free nutation 280-230 Ma
}

\author{
S. M. Molodensky \\ Schmidt Institute of Physics of the Earth, Russian Academy of Sciences, Moscow, Russia
}

\begin{abstract}
Recent paleomagnetic data have shown that the history of the Earth's magnetic field includes a unique, fairly long (280 Ma to $230 \mathrm{Ma}$ ) interval during which the orientation of the Earth's magnetic dipole remained stable relative to the Earth's rotation axis. As noted in [Molodensky, 1981], this geologic epoch coincided with the time when the retrograde annual nutation excited by the tidal wave $\Psi_{1}$ coincided in frequency with the nearly diurnal free resonance of the Earth's liquid core. The resonance excitation of the nearly diurnal free nutation could lead to an abrupt increase in the amplitude of nearly diurnal oscillations of the liquid core, and this could result in a drastic change in the geomagnetic dynamo regime (particularly in the turbulent boundary layer at the liquid core/mantle boundary, where the velocity gradient of the nearly diurnal oscillations is highest). To estimate the effectiveness of this mechanism, one should evidently take into account effects of tidal energy dissipation; due to these effects, the resonance amplitude tends toward a certain finite limit. For this purpose, below I address the resonance effects of the tidal energy absorption in (i) oceans, (ii) inelastic mantle, and (iii) viscous core with due regard for the electromagnetic coupling of the liquid core with the mantle. In order to estimate the inelasticity of the mantle in the range of tidal frequencies, the viscosity of the liquid core, and the core-mantle electromagnetic coupling, I used results derived from analysis of new data on amplitudes and phases of forced nutation. The amplitude of resonance oscillations of the liquid core was shown to exceed their contemporary amplitude by about an order of magnitude, so that the hypothesis under consideration appears to be rather plausible.
\end{abstract}

\section{Formulation of the Problem}

The phenomenon of nearly diurnal free nutation of a planet with an ellipsoidal homogeneous ideal incompressible liquid core and a solid shell was discovered and examined simultaneously and independently in 1909-1910 in classical works by Hough, Sludsky, and Poincare (e.g. see [Lamb, 1945; Poincare, 1910]). This phenomenon reduces to the fact that, as the frequency of the diurnal tidal wave approaches the resonance frequency equal to

$$
\sigma_{p}=-\omega\left(1+e_{\mathrm{liq}} A / A_{2}\right)
$$

Copyright 2004 by the Russian Journal of Earth Sciences.

Paper number TJE04164.

ISSN: $1681-1208$ (online)

The online version of this paper was published 12 December 2004. URL: http://rjes.wdcb.ru/v06/tje04164/tje04164.htm (here $\omega$ is the angular frequency of the Earth's diurnal rotation, $e_{\text {liq }}$ is the geometric flattening of the liquid core, and $A$ and $A_{2}$ are the equatorial moments of inertia of the whole Earth and the solid shell, respectively), the amplitude of the differential rotation of the liquid core relative to the shell increases unboundedly, which leads to an unbounded increase in the amplitude of forced nutation of the shell in space excited by this wave.

In the case of a more realistic model of the Earth including an elastic or inelastic mantle, a nonideal liquid core, and ocean, resonance amplitudes of forced nutation and tidal strain of the shell have finite values determined by the total amount of energy absorbed over the tidal cycle. Therefore, correct estimation of the resonance amplitudes should take into account not only the mantle elasticity effect on the value of $\sigma_{p}$ but also all main dissipative effects.

The nearly diurnal resonance of the liquid core, influencing significantly precise measurements of amplitudes of forced nutation and diurnal earth tides, has recently been 
studied rather extensively. Earth models fitting best the entire set of modern astrometric and tidal gravity data were constructed [Molodensky, 2004] in order to describe dynamic tidal effects of the real Earth including a heterogeneous compressible viscous electrically conductive liquid core, inner solid core, viscoelastic shell, and ocean. In particular, analysis of these models showed that the best fit between theoretical and observed nutation amplitudes can be attained with the following values of parameters:

$$
\begin{gathered}
e_{\text {liq }}=0.002736 \pm 0.000001 \\
K_{\mathrm{m}}=1.0435 \pm 0.0005 \\
e_{\mathrm{sol}}=0.0053 \pm 0.0002
\end{gathered}
$$

where $e_{\text {liq }}$ and $e_{\text {sol }}$ are the effective dynamic flattenings of the outer liquid and inner solid cores, respectively, and $K_{\mathrm{m}}$ is the dimensionless coefficient of effective inelasticity of the mantle defined as the ratio of the coefficient of mantle effective rigidity $\lambda_{2}$ (with respect to volume forces of tidal type) for diurnal oscillations to its value for oscillations at a period of $200 \mathrm{~s}$ (the PREM model was constructed for this period).

The value $e_{\text {liq }}=0.002736$ exceeds the hydrodynamic equilibrium dynamic flattening of the liquid core $e_{\text {hyd }}=0.00256$ by about $8 \%$, and the value $e_{\mathrm{sol}}=0.0053$ is greater than the equilibrium flattening of the inner solid core by about two times. As was shown in [Molodensky, 2001; Molodensky and Groten, 2001], these divergences can be related to rheological properties of the mantle (due to their effect, the contemporary flattening of the liquid core can be conformable to the daily rotation velocity existing at the time $280-230 \mathrm{Ma}$, when its value and the equatorial ratio of the centrifugal force to the gravitational force was higher), as well as to the effect of the electromagnetic coupling of the liquid core with the mantle and the inner solid core, which produces an additional force moment acting on the inner solid core in the same way as the moment arising due to the ellipticity of the solid and liquid cores. With electrical conductivity values characteristic of metals at temperatures and pressures of the Earth's core, the magnetic field diffusion time is many orders greater than the period of nutation-induced oscillations of the solid core relative to the liquid core, implying that both electromagnetic and inertial coupling does not lead to tidal energy dissipation and a phase shift of the moment of electromagnetic forces relative to the moment of hydrodynamic pressure forces. Therefore, the fact that $e_{\text {sol }}$ is significantly greater than the hydrostatic flattening of the inner solid core $e_{\mathrm{h}}$ does not indicate that the real (geometric) flattening of the solid core differs from $e_{\mathrm{h}}$ significantly.

In the recent epoch, the period of the nearly diurnal free nutation in space determined by values (2),

$$
T=2 \pi /\left(\omega+\sigma_{p}\right)
$$

amounts to 434 sidereal days, which differs significantly from the solar year. However, due to a higher ratio of the centrifugal force to the gravitational force in the interval 280 $230 \mathrm{Ma}$, hydrostatic flattenings of the liquid core and the mantle were also considerably higher. As is evident from formulas (1) and (3), the period of nearly diurnal free nutation decreases with an increase in $e_{\text {liq. }}$.
In the recent epoch, the day duration is known to increase by about 2 milliseconds per century (mainly due to dissipation of energy in shallow ocean areas). However, estimates for remote geologic epochs (based on calculations of cotidal maps showing the then-existing land-sea distributions) yield an appreciably larger value. If one assumes, in accordance with [Zharkov et al., 1996], that an increase in the day duration over the last 280-230 Myr lie within the range $\delta T_{\text {day }} \sim(4-6) \cdot 10^{3} \mathrm{~s}$ and the value of the liquid core-mantle electromagnetic coupling was nearly the same as presently, the period of nearly diurnal free nutation in that epoch (with $\delta T_{\text {day }} \sim 5 \cdot 10^{3} \mathrm{~s}$ ) should have been exactly equal to the solar year, and the frequency of retrograde annual nutation excited by the tidal wave $\Psi_{1}$ should have coincided with the nearly diurnal resonance frequency of the liquid core.

Resonance excitation of nearly diurnal free nutation abruptly increases the amplitude of nearly diurnal oscillations of the liquid core relative to the mantle. This should give rise to two observable effects: (1) a change in the geomagnetic dynamo regime (particularly in the turbulent boundary layer at liquid core/mantle interface, where the velocity gradient of nearly diurnal oscillations is highest) and (2) an abrupt increase in tidal strain amplitudes in the mantle (determined by the dimensionless Love ( $h$ and $k$ ) and Shida $(l)$ numbers.

The first effect can be associated with the period of anomalously rare changes in the orientation of the magnetic dipole relative to the Earth's geographic poles.

Movements of the Earth's magnetic poles (defined by the orientation of the dipole that characterizes the main part of the geomagnetic field) are reconstructed from paleomagnetic data. The movements of geomagnetic poles are most clearly determined from profile data on the geomagnetic field recorded across the strike of the Mid-Atlantic Ridge, where mantle material rises and, upon cooling, moves horizontally eastward and westward at an average velocity of about $2 \mathrm{~cm} \mathrm{yr}^{-1}$; during this process, rocks retain in their "memory" the magnetic field corresponding to the Curie point (the temperature at which ferromagnetic domains are pinned). Profile data obtained at various latitudes coincide, implying that the maximum distance between the geomagnetic and geographic poles never exceeded a few degrees. Polarity of the magnetic dipole experienced jumplike changes. The time of constant orientation of the magnetic dipole varies from a few tens of thousands to 50 million years (the longest period of the Earth's history during which the orientation did not change coincides with the interval 280$230 \mathrm{Ma})$. The northern magnetic pole was close to the northern geographic pole in about half of polarity change cases; the northern (southern) magnetic pole approximately coincides with the southern (northern) geographic pole in the other half of cases. The approximate coincidence of geographic and magnetic poles is accounted for by a strong effect of the Coriolis force on the convective motions in the liquid core. According to the Taylor-Proudman theorem (e.g. see [Greenspan, 1969]), stationary (geostrophic) flows of a rotating liquid meet the condition $\partial \mathbf{v} / \partial z=0$, where $\mathbf{v}$ is the velocity vector and the $z$ axis is oriented along the axis of the liquid rotation. On the strength of this relation, the so-called Taylor-Proudman columns oriented along the 
rotation axis arise in the rotating liquid [Greenspan, 1969]; motions in these columns are nearly axisymmetric and generate a dipole component of the magnetic field preferably oriented along the liquid rotation axis.

An abrupt rise in mantle amplitudes of the tidal strain in the resonance time interval can lead to observed effects (e.g. the effects that are associated with the rise in the amplitude of oceanic tides excited by the wave $\Psi_{1}$ and can be studied from data on daily and yearly nodes of fossil corals). Below, to obtain their estimates for the resonance epoch, I calculate the Love number $h$ (determining the ratios of vertical displacements of the Earth's surface to the vertical displacement of the equipotential surface of the summarized gravitational potential of the Earth and the tide-generating potential at its surface), the Love number $k$ (determining the ratio of the potential change due to the tidal mass redistribution inside the Earth to the tide-generating potential at its surface), and the Shida number $l$ (determining the horizontal tidal displacements of the Earth's surface).

\section{Description of the Model}

To estimate the effectiveness of the mechanism of resonance excitation of nearly diurnal free nutation, one should evidently take into account the effects of tidal energy dissipation due to which the resonance amplitude tends to a certain finite limit. For this purpose, resonance effects of tidal energy absorption are considered below (1) in oceans, (2) in an inelastic mantle, and (3) in a viscous core with regard for the electromagnetic coupling of the liquid core and mantle. The inelasticity of mantle in the tidal frequency range, the viscosity of the liquid core, and the core-mantle electromagnetic coupling were estimated with the use of new data on amplitudes and phases of forced nutation obtained in [Molodensky, 2000, 2004; Molodensky and Groten, 2001].

Estimates in an inelastic mantle were obtained with the use of the power-law function of creep with an exponent $\alpha$ [Smith and Dahlen, 1981]; in this case, the ratios of the real and imaginary parts of the shear modulus at frequencies $\omega$ and $\omega_{0}$ obey the relations (e.g. see [Zharkov et al., 1996])

$$
\begin{gathered}
\frac{\operatorname{Re} \mu(\omega)}{\operatorname{Re} \mu\left(\omega_{0}\right)}=1-\frac{f\left(\omega, \omega_{0}\right)}{Q_{\mu}\left(\omega_{0}, l\right)}, \\
\frac{\operatorname{Im} \mu(\omega)}{\operatorname{Im} \mu\left(\omega_{0}\right)}=g\left(\omega, \omega_{0}\right),
\end{gathered}
$$

where $Q_{\mu}$ are mechanical quality parameters, $l$ is the depth to a spherical layer, and $f\left(\omega, \omega_{0}\right)$ is a coefficient depending on rheological properties of the medium. In the particular case $\alpha \rightarrow 0$, this dependence includes the Lomnitz function of creep:

$$
\begin{gathered}
f\left(\omega, \omega_{0}\right)=\frac{2}{\pi} \ln \frac{\omega_{0}}{\omega} \\
g\left(\omega, \omega_{0}\right)=\text { const }
\end{gathered}
$$

In the more general case of a power function with $\alpha \neq 0$, the functions $f\left(\omega, \omega_{0}\right)$ and $g\left(\omega, \omega_{0}\right)$ are given by the expressions

$$
\begin{gathered}
f\left(\omega, \omega_{0}\right)=\cot \frac{n \pi}{2}\left(\left(\omega_{0} / \omega\right)^{n}-1\right) \\
g\left(\omega, \omega_{0}\right)=\left(\frac{\omega_{0}}{\omega}\right)^{n} .
\end{gathered}
$$

As was shown in [Molodensky, 2004], the optimal value of $\alpha$ in the range of tidal periods is $\alpha \sim 0.04$, and the quality factor $Q_{\mu}$ in the lower mantle (for the oscillation period $200 \mathrm{~s}$ ) is

$$
Q_{\mu} \sim 75
$$

The values of electromagnetic and viscous coupling between the liquid core and the shell are determined by the expressions [Molodensky, 2004]

$$
\begin{gathered}
\mathbf{T}_{e-m}=\iint|\xi| r_{c}\left(\mathbf{e}_{\vartheta} \dot{u}_{\varphi}^{\prime}-\mathbf{e}_{\varphi} \dot{u}_{\vartheta}^{\prime}\right) d S \\
=C_{c}\left[t_{\text {in }}^{(e-m)}\left(\mathbf{e}_{y} \cos \sigma t-\mathbf{e}_{x} \sin \sigma t\right)\right. \\
\left.+t_{\text {out }}^{(e-m)}\left(\mathbf{e}_{x} \cos \sigma t-\mathbf{e}_{y} \sin \sigma t\right)\right] ; \\
\mathbf{T}_{\mathrm{vis}}=\frac{\nu \sigma \widetilde{a} C_{c}}{\rho}\left[t_{\text {in }}^{(\mathrm{vis})}\left(\mathbf{e}_{y} \cos \sigma t-\mathbf{e}_{x} \sin \sigma t\right)\right. \\
\left.+t_{\text {out }}^{(\mathrm{vis})}\left(-\mathbf{e}_{x} \cos \sigma t-\mathbf{e}_{y} \sin \sigma t\right)\right],
\end{gathered}
$$

where

$$
\begin{gathered}
t_{\text {in }}^{(\mathrm{vis})}=\frac{15}{16} \int_{0}^{\pi}\left((1-\cos \vartheta)^{2} \widetilde{k}_{1}+(1+\cos \vartheta)^{2} \widetilde{k}_{2}\right) \sin \vartheta d \vartheta ; \\
t_{\text {out }}^{(\mathrm{vis})}=\frac{15}{16} \int_{0}^{\pi}\left((1-\cos \vartheta)^{2}\left|\widetilde{k}_{1}\right|+(1+\cos \vartheta)^{2}\left|\widetilde{k}_{2}\right|\right) \sin \vartheta d \vartheta ; \\
t_{\mathrm{in}}^{(e-m)}=\frac{15}{16} \int_{0}^{\pi}|\xi| \chi_{\mathrm{in}}(\vartheta) \sin \vartheta d \vartheta ; \\
t_{\mathrm{out}}^{(e-m)}=\frac{15}{16} \int_{0}^{\pi}|\xi| \chi_{\mathrm{out}}(\vartheta) \sin \vartheta d \vartheta ;
\end{gathered}
$$

$$
\chi_{\text {in }}(\vartheta)=\left\{\begin{array}{lll}
-\left|k_{2}\right|(\cos \vartheta+1)^{2} & \text { at } & \cos \vartheta \geq-f \\
0 & \text { at } & f<\cos \vartheta<-f \\
-\left|k_{1}\right|(\cos \vartheta-1)^{2} & \text { at } & \cos \vartheta \leq f
\end{array}\right.
$$

$\chi_{\text {out }}(\vartheta)=\left\{\begin{array}{l}\left|k_{1}\right|(\cos \vartheta-1)^{2} \text { at } \cos \vartheta \geq-f \\ \left|k_{1}\right|(\cos \vartheta-1)^{2}+\left|k_{2}\right|(\cos \vartheta+1)^{2} \\ \text { at } f<\cos \vartheta<-f ; \\ \left|k_{2}\right|(\cos \vartheta+1)^{2} \text { at } \cos \vartheta \leq f\end{array}\right.$ 
$\left|k_{1}\right|$ and $\left|k_{2}\right|$ are the moduli of roots of the quadratic equation

$$
\frac{\xi k^{2}}{\sigma^{2}}= \pm \frac{\cos \vartheta}{f}-1
$$

corresponding to different signs of its right-hand side and $f=\sigma /(2 \omega)$.

$$
\xi=\left(i \sigma \nu+H_{0}^{2} /(4 \pi)\right) / \rho
$$

$\nu$ is viscosity;

$$
\begin{gathered}
\widetilde{k}_{1}=\frac{\left|k_{1}\right|}{\sqrt{2}} \operatorname{sgn}(-\cos \vartheta+f), \\
\widetilde{k}_{2}=\frac{\left|k_{2}\right|}{\sqrt{2}} \operatorname{sgn}(\cos \vartheta+f),
\end{gathered}
$$

$H_{0}$ is the component of the magnetic field normal at the solid/liquid core and liquid core/mantle boundaries; and

$$
\operatorname{sgn}(x)=\left\{\begin{array}{c}
1, x \geq 0 \\
-1, x<0
\end{array}\right.
$$

is the signum function. Here $C_{c}$ is the principal moment of inertia of the liquid core and the indices "in" and "out" denote components of moments, respectively, coinciding in phase with the moments of the tidal forces (in-phase components) and lagging behind them by $\pi / 2$ (out-off-phase components).

Very few data are presently available on the angular distributions of $H_{0}$ in the core. However, since the moment of electromagnetic forces $C_{c} t_{\text {in }}^{(e-m)}\left(\mathbf{e}_{y} \cos \sigma t-\mathbf{e}_{x} \sin \sigma t\right)$ coincides in phase with the moments of tidal forces and forces of the hydrodynamic pressure acting on the solid/liquid core and liquid core/mantle ellipsoidal boundaries, the $H_{0}$ distributions can be taken into account by introducing parameters of the effective dynamic flattening of the solid and liquid cores. Because the coefficients $t_{\text {in }}^{(e-m)}$ determined by relation $(9 \mathrm{~b})$ are negative for any distributions of the functions $\xi(\vartheta, \varphi)(10 \mathrm{~b})$, the influence of this term reduces to the fact that the effective dynamic flattenings of the inner and outer cores $e_{\text {sol }}$ and $e_{\text {liq }}$ become greater than their geometric flattenings. Because of deficient data, I assume below that the values of the antiferromagnetic and viscous coupling did not significantly varied over the last $230 \mathrm{Myr}$ and, therefore, the deviations of $e_{\text {liq }}$ and $e_{\text {sol }}$ from their hydrostatic values remained constant.

The resonance excitation of nearly diurnal free nutation including effects of the mantle inelasticity and electromagnetic and viscous coupling between core and shell can be described in terms of the Liouville equation for the liquid core

$$
\dot{\mathbf{M}}+\boldsymbol{\omega} \times \mathbf{M}=\mathbf{L}=\mathbf{T}_{\mathrm{hyd}}+\mathbf{T}_{\mathrm{vis}}+\mathbf{T}_{e-m}
$$

where

$$
M_{i}=I_{i k} \omega_{k}+\int_{\tau} \rho(\mathbf{r} \times \mathbf{u})_{i} d \tau
$$

is the $i$ th component of the angular momentum of the liquid core, $I_{i k}$ is the product of its moments of inertia, $\tau$ is its volume, and $\mathbf{L}$ is the total moment of forces acting on the liquid core; the latter includes the moments of viscous $\left(\mathbf{T}_{\mathrm{vis}}\right)$, electromagnetic $\left(\mathbf{T}_{e-m}\right)$, and hydrodynamic pressure $\left(\mathbf{T}_{\text {hyd }}\right)$ forces.

In the bulk of the liquid core, I describe the displacement field by expressions of the simplest (Poincare) type:

$$
\begin{gathered}
\dot{\mathbf{u}}_{x}=\psi_{0} \frac{\omega^{2} z}{\sigma+2 \omega} \cos (\sigma t) ; \\
\dot{\mathbf{u}}_{y}=\psi_{0} \frac{\omega^{2} z}{\sigma+2 \omega} \sin (\sigma t) ; \\
\dot{\mathbf{u}}_{z}=\left(\Psi_{0} \omega^{2} / \sigma-2 \varepsilon \omega\right)(y \cos (\sigma t)-x \sin (\sigma t)),
\end{gathered}
$$

where $\psi_{0}$ is a dimensionless resonance parameter determining the nutation amplitude of the liquid core relative to the shell and $\varepsilon$ is the nutation amplitude relative to the moving (Tisserand) coordinate system. The substitution of (13) into (12) yields

$$
\begin{gathered}
\mathbf{M}=\left(\mathbf{e}_{x} \cos (\sigma t)+\mathbf{e}_{y} \sin (\sigma t)\right) \omega \\
\times\left\{\psi_{0}\left[\left(-A_{1}+C_{1} / 2\right) \frac{\omega}{\sigma+2 \omega}+\frac{C_{1} \omega}{2 \sigma}\right]-\varepsilon\left(C_{1}-A_{1}\right)+I_{1}\right\} \\
+C_{1} \omega \mathbf{e}_{z}=\left(\mathbf{e}_{x} \cos (\sigma t)+\mathbf{e}_{y} \sin (\sigma t)\right) C_{1} \omega \\
\times\left[\frac{\omega \psi_{0}}{\sigma+2 \omega}\left(\frac{\omega}{\sigma}+e_{d}\right)-\varepsilon e_{d}+\frac{I_{1}}{C_{1}}\right]+C_{1} \omega \mathbf{e}_{z},
\end{gathered}
$$

where $A_{1}$ and $C_{1}$ are the principal moments of inertia of the liquid core; $I_{1}$ is the variation amplitude of inertia products determined by the relations

$$
\begin{aligned}
& I_{x z}=-\iiint_{\tau} \rho x z d \tau=\operatorname{Re}\left(I_{1} e^{i \sigma t}\right) ; \\
& I_{y z}=-\iiint_{\tau} \rho y z d \tau=\operatorname{Im}\left(I_{1} e^{i \sigma t}\right),
\end{aligned}
$$

and $e_{\mathrm{d}}=\left(C_{1}-A_{1}\right) / C_{1}$ is the dynamic flattening of the liquid core.

In order to calculate the total moment of forces exerted by the liquid core on the shell, I calculate the moment of hydrodynamic pressure forces:

$$
\begin{gathered}
\mathbf{T}_{\mathrm{hyd}}=-\int_{\tau(t)} \rho \mathbf{r} \times \nabla \Psi d \tau \\
=\psi_{0} \omega^{2}\left[\left(C_{1}-A_{1}\right)\left(\mathbf{e}_{y} \cos (\sigma t)-\mathbf{e}_{x} \sin (\sigma t)\right)\right. \\
+I_{x y}\left(\mathbf{e}_{x} \cos (\sigma t)-\mathbf{e}_{y} \sin (\sigma t)\right) \\
\left.-\mathbf{e}_{z}\left(I_{y z} \cos (\sigma t)-I_{x z} \sin (\sigma t)\right)\right] .
\end{gathered}
$$


Table 1. Values of the amplitude ratio of forced nutation of the real Earth to nutation of the Earth's solid model ${ }^{\varepsilon / \varepsilon} 0$, the resonance parameter $\Psi_{0}$, and the Love numbers $h$ and $k$ and the Shida number $l$ calculated for dynamic flattenings of the liquid core $e_{\text {liq }}$ corresponding to various geologic epochs

\begin{tabular}{rrrrrr|rrrrrr}
\hline$e_{\text {liq }} \times 10^{3}$ & $\varepsilon / \varepsilon$ & $\Psi_{0} \times 10^{-3}$ & $h$ & $k$ & $l$ & $e_{\text {liq }} \times 10^{3}$ & $\varepsilon / \varepsilon 0$ & $\Psi_{0} \times 10^{-3}$ & $h$ & $k$ \\
\hline 2.736 & 1.390 & 1.249 & 1.148 & 0.559 & 0.063 & 3.052 & 14.4 & 43.11 & 19.2 & 9.3 & -0.617 \\
2.762 & 1.423 & 1.353 & 1.193 & 0.580 & 0.062 & 3.054 & 18.1 & 54.79 & 24.3 & 11.7 & -0.806 \\
2.788 & 1.461 & 1.477 & 1.246 & 0.606 & 0.060 & 3.055 & 20.7 & 63.37 & 28.0 & 13.5 & -0.946 \\
2.813 & 1.507 & 1.625 & 1.311 & 0.637 & 0.057 & 3.056 & 24.4 & 75.14 & 33.1 & 15.9 & -1.137 \\
2.838 & 1.564 & 1.806 & 1.389 & 0.675 & 0.054 & 3.057 & 29.7 & 92.29 & 40.5 & 19.5 & -1.416 \\
2.850 & 1.597 & 1.913 & 1.435 & 0.697 & 0.052 & 3.058 & 38.2 & 119.5 & 52.2 & 25.2 & -1.859 \\
2.862 & 1.635 & 2.034 & 1.487 & 0.722 & 0.051 & 3.059 & 53.9 & 169.7 & 73.9 & 35.6 & -2.674 \\
2.875 & 1.677 & 2.170 & 1.546 & 0.750 & 0.048 & 3.060 & 92.1 & 292.4 & 126.9 & 61.1 & -4.667 \\
2.887 & 1.726 & 2.326 & 1.613 & 0.783 & 0.046 & 3.061 & 128 & 405.6 & 156.6 & 75.8 & -5.371 \\
2.900 & 1.782 & 2.506 & 1.691 & 0.820 & 0.043 & 3.062 & -103 & -309.6 & -142.5 & -69.0 & 4.933 \\
2.913 & 1.847 & 2.717 & 1.782 & 0.864 & 0.039 & 3.063 & -76.9 & -250.1 & -107.4 & -51.7 & 4.147 \\
2.925 & 1.925 & 2.966 & 1.889 & 0.916 & 0.035 & 3.064 & -47.1 & -154.5 & -66.1 & -31.8 & 2.594 \\
2.938 & 2.018 & 3.265 & 2.019 & 0.978 & 0.031 & 3.065 & -33.8 & -111.8 & -47.7 & -22.9 & 1.900 \\
2.950 & 2.132 & 3.631 & 2.177 & 1.054 & 0.025 & 3.066 & -26.3 & -87.62 & -37.2 & -17.9 & 1.507 \\
2.963 & 2.275 & 4.091 & 2.375 & 1.149 & 0.017 & 3.067 & -21.4 & -72.02 & -30.5 & -14.7 & 1.253 \\
2.975 & 2.460 & 4.683 & 2.631 & 1.272 & 0.008 & 3.069 & -15.5 & -53.11 & -22.3 & -10.7 & 0.946 \\
2.987 & 2.706 & 5.475 & 2.973 & 1.437 & -0.005 & 3.071 & -12.1 & -42.06 & -17.6 & -8.4 & 0.767 \\
3.000 & 3.053 & 6.590 & 3.454 & 1.669 & -0.023 & 3.073 & -9.8 & -34.82 & -14.4 & -6.9 & 0.649 \\
3.012 & 3.578 & 8.275 & 4.182 & 2.019 & -0.051 & 3.075 & -8.3 & -29.70 & -12.2 & -5.9 & 0.566 \\
3.025 & 4.464 & 11.11 & 5.409 & 2.610 & -0.097 & 3.100 & -2.2 & -10.47 & -3.91 & -1.878 & 0.254 \\
3.037 & 6.276 & 16.93 & 7.921 & 3.819 & -0.192 & 3.150 & -0.42 & -4.564 & -1.36 & -0.650 & 0.158 \\
3.050 & 12.1 & 35.53 & 15.9 & 7.685 & -0.494 & 3.200 & 0.092 & -2.918 & -0.651 & -0.308 & 0.131 \\
\hline
\end{tabular}

The substitution of relations (8a), (8b), (14) and (17) into (11) yields

$$
\begin{aligned}
& \psi_{0}\left(\frac{\omega(\sigma+\omega)}{\sigma(\sigma+2 \omega)}-\widetilde{e}_{d} \frac{\omega}{\sigma+2 \omega}\right) \\
& -\varepsilon\left(1+\widetilde{e}_{d} \frac{\sigma+\omega}{\omega}\right)+\frac{\sigma}{\omega} \frac{I_{1}}{C_{1}}=0,
\end{aligned}
$$

where

$$
\widetilde{e}_{d}=e_{d}+t_{\mathrm{in}}^{(e-m)}+\frac{\nu \sigma \widetilde{a}}{\rho} t_{\mathrm{in}}^{(\mathrm{vis})}+i\left(t_{\mathrm{in}}^{(e-m)}+\frac{\nu \sigma \widetilde{a}}{\rho} t_{\mathrm{in}}^{(\mathrm{vis})}\right)
$$

- is the effective dynamic flattening of the core/mantle boundary.

The second relation connecting the parameters $\psi_{0}$ and $\varepsilon$ can be obtained by using the continuity condition for the normal component of displacements at the core/mantle ellipsoidal boundary. The normal to this surface can be represented as

$$
\mathbf{n}=\left(\mathbf{e}_{r}+2 e_{g} \mathbf{e}_{z} \cos \vartheta\right)\left(1-2 e_{g} \cos ^{2} \vartheta\right)
$$

where $e_{g}$ is the geometric flattening of this surface. In the case of diurnal tidal waves, the dependence of the normal component of displacements of the core/mantle inelastic boundary on the angular variables $(\vartheta, \phi)$ and time $t$ can be represented as

$$
U_{n}^{(m)}=h^{(m)} \sin \vartheta \cos \vartheta \cos (\sigma t-\phi)
$$

where $h^{(m)}$ is the complex amplitude independent of $(\vartheta, \phi, t)$. Then, I obtain

$$
\begin{aligned}
& \psi_{0}^{(m)}\left(\frac{\omega^{2}(\sigma+\omega)}{\sigma^{2}(\sigma+2 \omega)}+e_{g} \frac{\omega^{2}}{\sigma^{2}}\right) \\
& -\varepsilon\left(\frac{\omega}{\sigma}\left(1+2 e_{g}\right)\right)-\frac{h^{(m)}}{2 r_{0}}\left(1-\frac{12}{7} e_{g}\right)=0 .
\end{aligned}
$$

Equations (18) and (20) form a closed system of two algebraic equations (with complex coefficients) with respect to the two unknown complex parameters $\varepsilon$ and $\psi_{0}$ determining amplitudes and phases of the shell nutation and tidal oscillations of the core relative to the shell near the resonance. The values of $I_{1}$ and $h^{(m)}$ represented as coefficients in these equations were calculated for rheological models (4)-(6) in [Molodensky, 2004].

Below, I demonstrate that, with these values of parameters, the amplitude of resonance oscillations of the liquid core is about one and a half order of magnitude higher than their amplitude in the recent epoch and, consequently, the hypothesis that the geomagnetic variation pattern and the resonance excitation of nearly diurnal nutation were interrelated in the geologic epoch under consideration is fairly well substantiated. 


\section{Numerical Results}

For the numerical simulation of the resonance excitation of nearly diurnal free nutation in the interval 280-230 Ma, I used values of viscosity of the core and quality factor of the mantle from admissible ranges estimated in [Molodensky, 2002, 2004]. Because of lacking data on the temporal variation in the core-mantle electromagnetic coupling, I used its contemporary value determined by the real part of the contemporary dynamic flattening of the liquid core (see formula (2)).

Amplitude ratios of forced nutation of the real Earth to nutation of the Earth's solid model ${ }^{\varepsilon / \varepsilon} 0$, resonance parameters $\Psi_{0}$, and dynamic values of the Love numbers $h$ and $k$ and the Shida number $l$ calculated by formulas (18) and (20) are presented in the Table 1 for geologic epochs corresponding to various values of the dynamic flattening of the liquid core $e_{\text {liq }}$. The first value $e_{\text {liq }}=2.736 \times 10^{-3}$, presented in this table, is the effective dynamic flattening of the liquid core (including its electromagnetic coupling with the mantle) in the recent epoch, whereas the value $e_{\text {liq }}=3.0615 \times 10^{-3}$ corresponds to an epoch of approximately 280-230 Ma (more accurate dates are not presented due to determination uncertainties in values of the secular slowing-down of the Earth's daily rotation, although one may evidently assume that, in the first approximation, $e_{\text {liq }}$ decreases linearly with time).

As seen from the table, the Love and Shida numbers of the resonance epoch exceed their contemporary values by about two orders of magnitude, and resonance values of the factor $\Psi_{0}$ (proportional to the amplitude of diurnal oscillations of the liquid core relative to the shell) are about 300 times greater than its contemporary values for the same retrograde annual component. The values of $\Psi_{0}$ are about an order of magnitude higher than the amplitude of contemporary nearly diurnal oscillations of the core (excited by the main lunisolar component $K_{1}$ ). Taking into account that, given turbulent friction of the core against the shell, the force of viscous friction is proportional to the second power of the resonance factor, and the absorbed energy is proportional to its third power, it is evident that the dissipation rate of tidal energy at the core/mantle boundary should have increased by a about $10^{3}$ times. Therefore, the hypothesis on the interrelation between the geomagnetic variation pat- tern and the resonance excitation of nearly diurnal nutation 280-230 Ma is fairly plausible. Moreover, the estimate of the contribution of this effect to the history of the Earth's thermal balance is of significant interest.

Acknowledgments. This work was supported by the Division of Earth Sciences, Russian Academy of Sciences, program 5 "Interaction of a Plume with the Lithosphere."

\section{References}

Greenspan, H. P. (1969), The Theory of Rotating Fluids, p. 467, Cambridge University Press.

Lamb, H. (1945), Hydrodynamics, p. 738, Dover, New York.

Molodensky, S. M. (1981), The influence of ocean and viscosity of the shell on the Earth's nutation, Fizika Zemli, (4), 17-24.

Molodensky, S. M. (2000), Viscosity effects of the liquid core in the theory of the Earth's nutation, Izv., Phys. Solid Earth, 36, $114-120$.

Molodensky, S. M. (2001), Lower mantle viscosity constraints from earth tides, nutation, and secular deceleration of rotation, Izv., Phys. Solid Earth, 37, 529-535.

Molodensky, S. M. (2004), Effect of the Electromagnetic CoreMantle Coupling on the Earth's Nutation, 2. Comparison with Observations, Izv., Phys. Solid Earth, 40(9), 3-14.

Molodensky, S. M., and E. Groten (1997), On atmospheric excitation of out-of-phase nutational components, Astron. Astrophys., 327, 800-812.

Molodensky, S. M., and E. Groten (1998), On the dynamical effects of the inhomogeneous liquid core in the theory of Chandler wobble, Geophys. J. Int., 135, 723-726.

Molodensky, S. M., and E. Groten (2001), On the models of the lower mantle viscosity consistent with the modern data of coremantle boundary flattening, Stud. Geophys. Geod., 45, 12-36.

Poincare, H. (1910), Sur la precession des corps deformables, Bull. Astron., 27, 321-356.

Smith, M. L., and F. A. Dahlen (1981), The period and $Q$ of the Chandler wobble, Geophys. J. R. Astron. Soc., 64, 223-284.

Zharkov, V. N., S. M. Molodensky, A. Brzezinski, E. Groten, and P. Varga (1996), The Earth and Its Rotation, Low Frequency Geodynamics, p. 508, Herbert Wichmann Verlag, Heidelberg.

S. M. Molodensky, Schmidt Institute of Physics of the Earth, Russian Academy of Sciences, 10, Bol'shaya Gruzinskaya ul., Moscow, 123995 Russia

(Received 24 October 2004) 